\title{
Nonlinear Model Predictive Control of Functional Electrical Stimulation
}

\author{
Nicholas Kirsch, Naji Alibeji, and Nitin Sharma ${ }^{1}$ \\ Department of Mechanical Engineering and Materials Science, University of Pittsburgh, \\ Pittsburgh, PA,USA 15261.
}

\begin{abstract}
Minimizing the amount of electrical stimulation can potentially mitigate the adverse effects of muscle fatigue during functional electrical stimulation (FES) induced limb movements. A gradient projection-based model predictive controller is presented for optimal control of a knee extension elicited via FES. A control Lyapunov function was used as a terminal cost to ensure stability of the model predictive control. The controller validation results show that the algorithm can be implemented in real-time with a steady-state RMS error of less than $2^{\circ}$. The experiments also show that the controller follows step changes in desired angles and is robust to external disturbances.
\end{abstract}

Keywords: Functional Electrical Stimulation, Nonlinear Model Predictive Control, Muscle Parameter Identification, Rehabilitation Engineering, Gradient Projection Algorithm

\section{Introduction}

An upper motor neuron disease or disorder impairs an individual's ability to perform functional movements, such as standing, walking, reaching, and grasping. Functional electrical stimulation (FES) is the application of low-level electrical current to the nerves that innervate the muscles to cause functional limb motion. FES has the potential to restore limb movements in individuals with corticospinal impairments. For example, by stimulating specific muscle groups in an appropriate sequential manner a walking motion can be achieved $[3,21,24,30,31,33]$. Most FES devices, such as the Parastep system [29] (Therapeutics Inc.), use electrodes placed on the surface of the skin (transcutaneous electrodes) to enable paraplegics to achieve standing and walking. However, this causes the muscles to fatigue more rapidly than normal, volitional muscle contractions. Muscle fatigue is the decline in the ability of a muscle to

Email address: \{nak65;naa33;nis62\}@pitt.edu (Nicholas Kirsch, Naji Alibeji, and Nitin Sharma)

${ }^{1}$ Corresponding Author; email: nis62@pitt.edu; fax:412-624-4846 
produce a force, and typically occurs due to fatigue of the nervous system or metabolic fatigue. In the case of transcutaneous electrical stimulation, the manner in which the muscle fibers are recruited differs from how muscle fibers are recruited during a natural, volitional contraction. This difference causes FESinduced muscle fatigue to occur at a significantly more rapid rate. There are two theories as to how the muscle fibers are recruited due to the application of FES, and why it causes rapid muscle fatigue. The first theory is that the muscle fibers are recruited in an inverse manner of the Henneman's size principle [35], in other words FES induced contractions recruit the motor units with larger motor axons (large force, fatigue rapidly) more easily than the motor units with smaller motor axons (low force, fatigue resistant). Because, the larger force producing and fatiguing muscle fibers are recruited with relative ease, the fatigue sets in rapidly. The second theory is that FES inherently recruits muscle fibers in a repeated, spatially fixed, and non-selective manner [5], which means that transcutaneous FES has no control over the motor units that are recruited. Because the same muscle fibers are recruited repeatedly rapid muscle fatigue occurs. Regardless of which of theses theories is correct, the rapid onset of muscle fatigue greatly limits the duration for which FES-based devices can be used. Error-based feedback control of FES [1, 2, 44, 46] can compensate for lower force production, due to muscle fatigue, by increasing the amplitude or frequency of electrical stimulation. However, increasing amplitude or frequency of stimulation can further aggravate the rate at which muscle fatigue occurs.

Recent advances in hybrid powered walking orthosis [16, 17], or use of orthosis or an exoskeleton [13] in general, can reduce stimulation duty cycle of FES because orthosis can be used to share or reduce stimulation of certain muscles during walking. However, stimulation of muscles when orthosis is not employed (e.g., during swing phase of FES + passive orthosis-based walking [45]) or during shared control between a powered orthosis and FES [8, 22, 23, 38, 39] is still a significant problem. Optimal control techniques can be used to produce the minimum amount of stimulation that is required to create a desired limb motion, thus reducing muscle fatigue. In $[37,45]$ optimizations of musculoskeletal gait models were used to compute the minimum amount of stimulation required to produce a gait motion. The computed stimulation patterns can be applied in open-loop control to reproduce the desired gait. However, open-loop optimal control techniques are not robust to disturbances or modeling errors due to the lack of feedback. In [49] a PD controller with an adaptive inverse optimal controller was used to control knee extension through FES. This robust technique incorporates error-based feedback control with a neural network that compensates for uncertainties in the musculoskeletal model. However, this technique did not solve the optimal control problem of an a priori cost function.

Unlike inverse optimal control, model predictive control (MPC) can solve the optimal control problem given an a priori cost function. Also, unlike open-loop optimal control techniques, MPC uses feedback that makes it more robust to disturbances. An optimal control-based controller when implemented runs in open loop because control inputs are computed for infinite horizon. MPC (also known as receding horizon control) uses a mathematical model of a system to 
predict how it will behave over a finite time horizon. Then by minimizing a userdefined cost function, control signals over the finite time horizon are numerically computed. The current state of the system is measured at each discrete time step of the control, which MPC uses as an initial condition for the next horizon. These initial conditions also act as feedback for the control. However, only the first element of the computed optimal control sequence is implemented on the system. In the next iteration the measured state is updated, the prediction horizon is shifted one time step forward, and the procedure is repeated.

MPC has been proposed for the control of FES for FES-assisted standing in [15] and for drop foot correction in [4]. In [15] MPC was simulated on a musculoskeletal model of the lower extremities and torso to track trajectories that minimize joint torque and jerk, enabling the model to make a sit-to-stand transfer. In [4] MPC was used in simulations on a musculoskeletal model to compute stimulation to the tibialis anterior muscle that minimizes stimulation and ground clearance of the foot during a step. The nonlinear dynamics of the musculoskeletal system and time-varying muscular response to FES makes MPC of a musculoskeletal system challenging. In [36] MPC was coupled with an input-output feedback linearization controller and simulated on FES control of knee extension. The input-output feedback linearization controller was used to cancel out the nonlinear dynamics of the musculoskeletal systems, thus making the optimal control problem simpler to solve. MPC was then used to control the linearized system instead of the nonlinear system, which resulted in computation times less than $20 \mathrm{~ms}$ in simulations. Therefore, the controller developed in [36] can potentially be applied for real-time MPC of FES with a control frequency of no more than $50 \mathrm{~Hz}$. However, its experimental verification remains to be seen.

In this paper, a nonlinear MPC (NMPC) algorithm that controls a nonlinear musculoskeletal system driven via FES is presented. The work presented in this paper is an expansion on the results presented in [27], which only presented pre-

liminary results for one participant. A gradient projection method was used to solve the optimal control problem, which has sufficiently fast computation times to facilitate real-time implementation of NMPC [19] and [25]. This paper also presents a musculoskeletal model with muscle activation dynamics and a procedure for estimating the subject specific parameters of the model that can be used for the implementation of NMPC of FES for knee extensions. Simulations and experimental results obtained from 3 able-bodied individuals illustrated that the NMPC method can be used to control knee extension via FES with approximately $2^{\underline{0}}$ of steady-state RMS error. The NMPC algorithm was also shown to be robust to impulsive disturbances during the knee regulation experiments. Because NMPC is an optimal control technique it may reduce the amount of stimulation required to produce a desired motion, thus reducing the effects of FES-induced muscle fatigue. Potentially, the proposed NMPC method may benefit FES-based gait restoration devices by increasing walking durations.

\section{Leg Extension Neuroprosthesis Model}

The leg extension dynamics during FES can be described as 


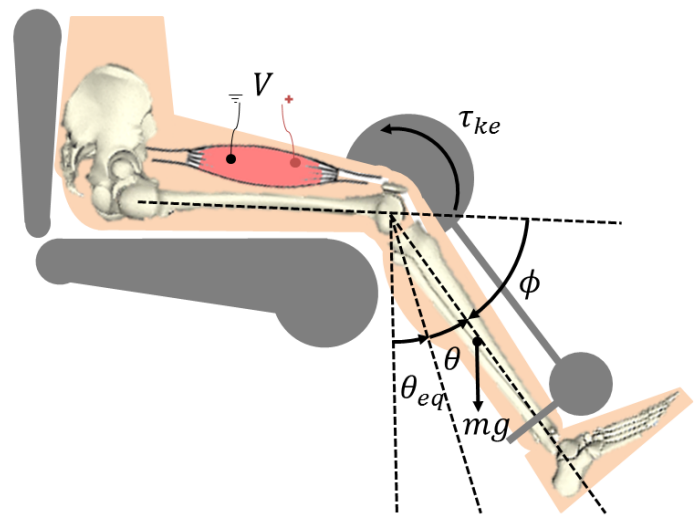

Figure 1: This knee extension neuroprosthesis uses electrical stimulation of the quadriceps muscles to elicit a knee extension. The angle $\theta$ is the angle of the lower leg relative to the equilibrium position of the lower leg, and the angle $\phi$ is the anatomical knee joint angle.

$$
J \ddot{\theta}+G-\tau_{p}=\tau_{k e},
$$

where $\theta, \dot{\theta}, \ddot{\theta} \in \mathbb{R}$ are the angular position, velocity, and acceleration of the lower leg (shank and foot) relative to equilibrium as illustrated in Fig. $1, J$ is the moment of inertia of the lower leg, and $G(\theta)=m g l_{c} \sin \left(\theta+\theta_{e q}\right)$ is the gravitational torque. In the gravitational torque $m$ is the mass of the lower leg, $g$ is gravitational acceleration, $l_{c}$ is the distance from the knee joint to the center of mass, and $\theta_{e q}$ is the equilibrium position of the lower leg relative to vertical as illustrated in Fig. 1. The passive musculoskeletal torque of the knee joint, $\tau_{p}(\theta, \dot{\theta})$ in $(1)$, is modeled as

$$
\tau_{p}=d_{1}\left(\phi-\phi_{0}\right)+d_{2} \dot{\phi}+d_{3} \mathrm{e}^{d_{4} \phi}-d_{5} \mathrm{e}^{d_{6} \phi},
$$

where the anatomical knee joint angle and angular velocity, $\phi, \dot{\phi} \in \mathbb{R}$, are defined as $\phi=\frac{\pi}{2}-\theta-\theta_{e q}$ and $\dot{\phi}=-\dot{\theta}$. The parameters $d_{i}(i=[1-6])$ and $\phi_{0}$ are subject specific parameters that model the stiffness and damping of the knee joint. The exponential terms in $\tau_{p}$ model hyperextension and hyperflexion of the knee joint.

The torque produced by the muscles due to an FES induced muscle contraction, $\tau_{k e}\left(\theta, \dot{\theta}, a_{k e}\right)$, is modeled using torque-length and torque-velocity muscle relationships as

$$
\tau_{k e}=\left(c_{2} \phi^{2}+c_{1} \phi+c_{0}\right)\left(1+c_{3} \dot{\phi}\right) a_{k e},
$$

where $c_{j}(j=[0-3])$ are subject specific parameters. The passive and active knee joint torques are based on Hill-type muscle models in[37]. Muscle activation, $a_{k e} \in[0,1]$, can be modeled as a first order system [48] as 


$$
\dot{a}_{k e}=\frac{u_{k e}-a_{k e}}{T_{a}},
$$

where $u_{k e} \in[0,1]$ is the normalized electrical stimulation amplitude and $T_{a} \in \mathbb{R}$ is the time constant of muscle activation. The normalized electrical stimulation amplitude can be mapped to the current amplitude of the electrical stimulation $[42,48]$ as

$$
I=I_{t}+u_{k e}\left(I_{s}-I_{t}\right),
$$

where $I_{t}$ is the minimum current amplitude required to produce a movement (threshold) and $I_{s}$ is the minimum current amplitude that produces the maximum muscle force (saturation).

The threshold and saturation current amplitudes are subject specific, and vary depending on the muscle being stimulated and the parameters of the stimulation train. Without loss of generality, $u_{k e}$ is mapped to the stimulation current amplitude, I. A similar relationship can be found for stimulation voltage amplitude and pulsewidth control of FES.

To implement the NMPC algorithm the dynamics in (1) and (4) are expressed in a state-space formulation as

$$
\begin{aligned}
\dot{x} & =f(x, u) \\
& =\left[\begin{array}{c}
x_{2} \\
-\beta \sin \left(x_{1}+\theta_{e q}\right)+\alpha\left(\tau_{p}+\tau_{k e}\right) \\
\frac{u-x_{3}}{T_{a}}
\end{array}\right],
\end{aligned}
$$

where $x=\left[\theta, \dot{\theta}, a_{k e}\right]^{T}, u=u_{k e}, \alpha=1 / J, \beta=m g l_{c} \alpha$, and $\tau_{k e}(x)$ can be determined from (3) and the relationship $\phi=\frac{\pi}{2}-x_{1}-\theta_{e q}$. To implement the gradient projection algorithm it is necessary that the state-space dynamics be continuously differentiable. From (6), it is clear that $f(x, u)$ is in fact continuously differentiable. Note that in this paper it is assumed that the dynamics of the system are time-invariant. When the effects of muscle fatigue become more prominent this assumption may become invalid (e.g. muscle fatigue affects the muscle recruitment curve). For this paper time-invariance will be assumed; however, future work will include muscle fatigue dynamics as modeled in [40] to make the MPC more robust to fatigue.

\section{Parameter Estimation}

To implement MPC, the subject specific parameters in (6) need to be estimated. This section describes procedures, which are based on methods in [47] and [40], for estimating these parameters. Results of each part of the parameter estimation procedure will be shown for Participant 1 to exemplify each step of the procedure. Three able bodied persons participated in the study. Each participant was instructed to remain relaxed and not influence any of the 


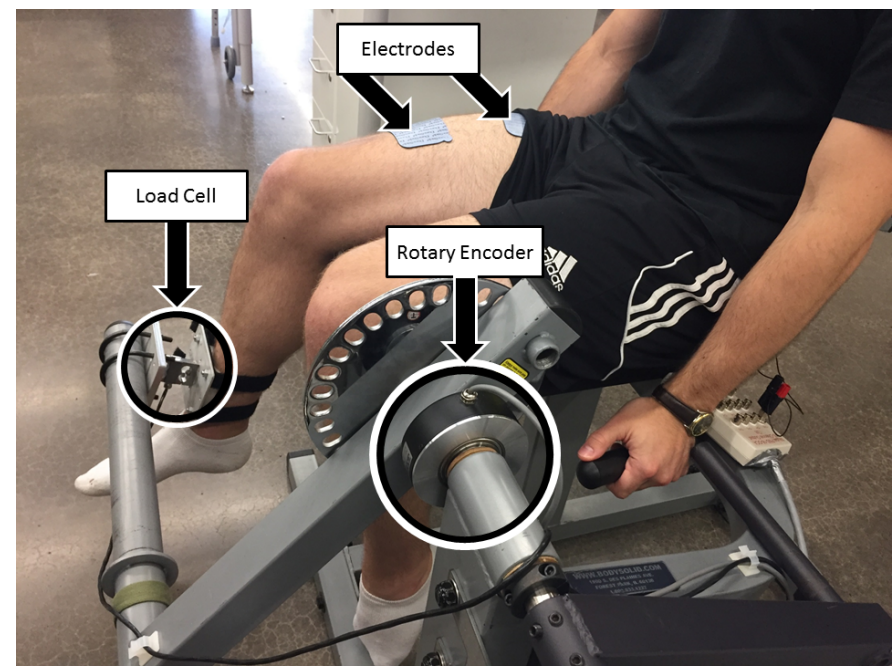

Figure 2: Participants were seated in a leg extension machine that has been modified with an encoder and load cell. Electrical stimulation of the quadriceps muscles was used to produced the knee extension motion.

measurements with volitional contractions during the experiments. For the experiments a bipolar, $35 \mathrm{~Hz}$ pulse train with a pulse width of $400 \mu \mathrm{s}$ was used. Five procedures were performed on the participants to identify their model parameters. The order in which the tests were performed is the same as they are listed below. The order that the procedures is important because

Test 1. The saturation and threshold current amplitudes $\left(I_{s}\right.$ and $I_{t}$ in (5)) were estimated. With the participant seated in an isometric contraction configuration, 2 second long pulse trains of stimulation were administered and the joint torque was measured using a load cell, as shown in Fig. 2. The stimulation current amplitude of the two second long pulse trains was slowly increased from $20-80 \mathrm{~mA}$, with two seconds in between the pulse trains. Only twenty different current amplitudes, evenly spaced from $20-80 \mathrm{~mA}$, and 2 second long pulse trains are used instead of a continuous current ramp so that the muscles are not unnecessarily fatigued. The peak current amplitude was set to be $80 \mathrm{~mA}$ because participants found current amplitudes above that level to be uncomfortable. The threshold current amplitude $\left(I_{t}\right)$ of an individual was taken as the current amplitude that produced the first significant muscle contraction. Increases in the current amplitude that no longer produce a significant increase in the torque were considered to be above the saturation limit. Therefore, the amplitude of the current pulse that produced the last significant change in torque is the saturation current amplitude $\left(I_{s}\right)$ of that individual. The torque measurements are plotted with the current amplitudes against time in Fig. 3. From this data the threshold was determined to be $23.2 \mathrm{~mA}$ (the second current pulse), and the saturation was determined to be $76.8 \mathrm{~mA}$ (the second to last current pulse). These values were determined automatically for each subject. The threshold 


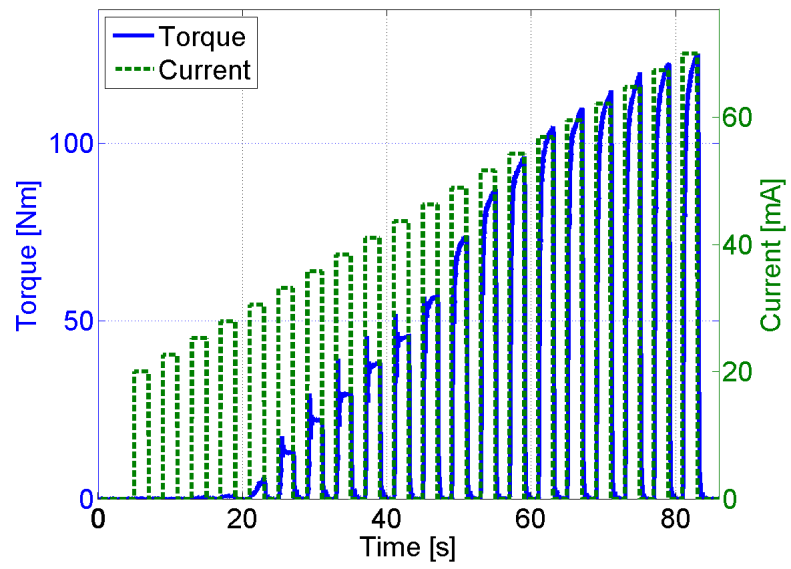

Figure 3: Stimulation current amplitude ramp to determine the saturation and threshold of the participant. The threshold is the current amplitude that causes the first significant torque measurement, and the saturation is the current amplitude that produced the last significant change in the torque measurement.

was computed as the stimulation amplitude that produces a load cell measurement three times greater than the variance of the load cell, and the saturation is the first stimulation amplitude that produced a torque greater than $99 \%$ of the maximum torque that was measured.

Test 2. The passive stiffness parameters $\left(d_{i}\right.$ for $i=[1,3,4,5,6]$ and $\theta_{e q}$ in $(2))$ and the mass parameters ( $m$ and $l_{c}$ in (1)) were determined by holding the participant's leg in different joint positions with a load cell, which was used to measure the passive knee joint torque at that position. Because no stimulation was used during this procedure and because the leg is stationary parameters corresponding to torque-length/velocity, muscle activation, inertia, and muscle damping may be ignored. A nonlinear, least-squares curve fitting algorithm was used to determine parameters that resulted in a best fit between the measured data and the function of the passive knee torque, $\tau_{p}$. The measured data and the resulting best fit determined by the nonlinear, least-squares curve fitting algorithm are shown in Fig. 4.

Test 3. Torque-angle parameters $\left(c_{i}\right.$ for $i=[0-2]$ in $\left.(3)\right)$ and the activation time constant $\left(T_{a}\right.$ in $\left.(4)\right)$ were determined from isometric contraction data while stimulating at the saturation level (determined from Test 1) for two seconds at a number of different joint angles. Because the leg is stationary during this test the parameters corresponding to torque-velocity, inertia, and muscle damping may be ignored. Also, by stimulating the muscle for two seconds and using the maximum torque measured as the maximum isometric contraction the muscle activation dynamics can be ignored. Like Test 2 a nonlinear, least squares curve fitting algorithm was used to determine torque-angle parameters that resulted in a best fit between the measured torque and joint angle at the 


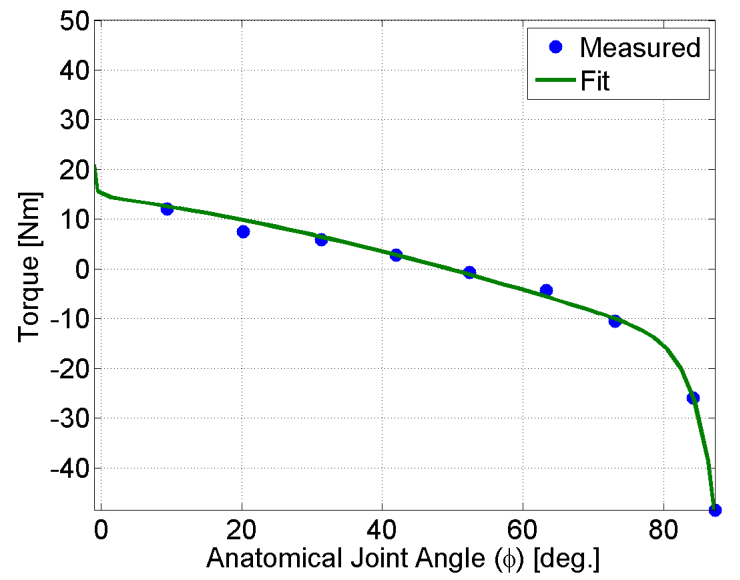

Figure 4: Push/pull tests to determine the mass and stiffness parameters of the musculoskeletal system. The exponential terms that model hyperextension and hyperflexion of the anatomical joint angles $(\phi)$ can be observed around $0^{0}$ and $85^{\circ}$, respectively.

different positions. The isometric contraction torques were measured at seven different joint positions and the best fit to the measured data are shown in Fig. 5 .

One of the isometric contraction tests was used to determine the muscle activation time constant that best matches the first order time response measured by the load cell. The muscle was stimulated at the saturation level, which corresponds to a normalized stimulation of 1 , and the joint torque was measured using the load cell. Under these conditions the normalized load cell measurement is a good approximation of the muscle activation because it was assumed that the muscle activation is equal to the normalized joint torque. The normalized load cell data from an isometric contraction is shown in Fig. 6, where the stimulation begins at one second. The muscle activation time constant of the first order response in (4) that best fits the measured data was determined to 0.18 seconds.

Test 4. The damping and inertial parameters of the system $\left(d_{2}\right.$ in $(2)$ and $J$ in (1)) were determined by a pendulum test. This was done by holding the leg at approximately full extension, then releasing it and allowing it to fall. An optical encoder in the leg extension machine at the knee joint, shown in Fig. 1, was used to measure the decaying, oscillating response of the leg as it falls. Because no stimulation is used during this procedure parameters corresponding to torquelength/velocity and muscle activation may be ignored. Then an optimization was then used to determine the damping and inertial parameters that result in a model that best fits the measured data. The measured encoder data from the pendulum test and the response from the best fit model are shown together in Fig. 7. Some discrepancy between the measured data and fit occur due to how the parameters that were previously determined in Test 2, which are 


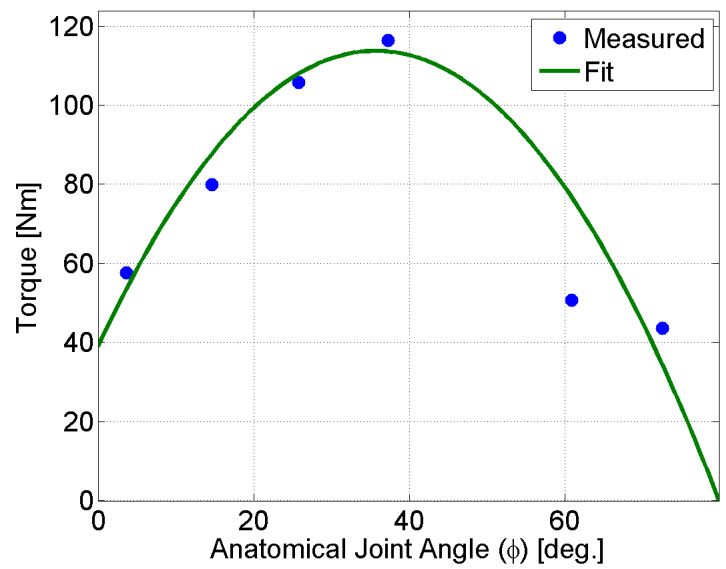

Figure 5: Torques produced during isometric contraction tests at different anatomical joint angles $(\phi)$, and the best fit of the force-length model to the measured data.

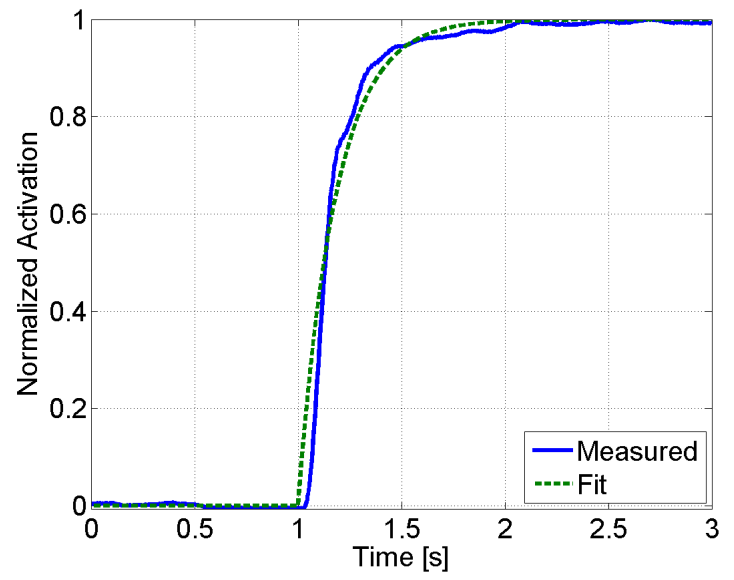

Figure 6: For an isometric contraction produced by a stimulation at the saturation current amplitude the normalized joint torque is approximately equal to muscle activation. Therefore, the load cell data can be used to determine the muscle activation time constant by determining the first order system time constant that results in a response that best fits the normalized data measured from the load cell. 


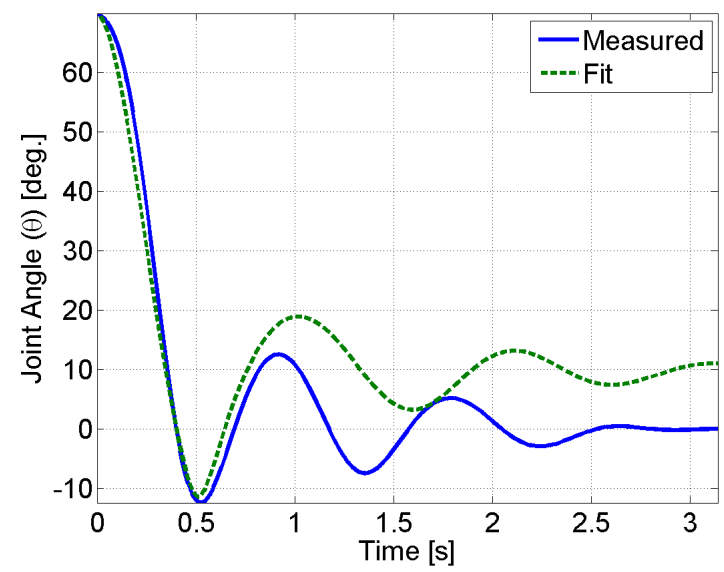

Figure 7: Plot of the results of the pendulum test with the response of the model that best fits the measured response.

kept constant during this estimation, affect the dynamics in a pendulum test. Therefore, it is important to note that errors in determining the parameters in Test 2 may result in errors in estimating the parameters in this test.

Test 5. A sinusoidal stimulation, with a period of 8 seconds, was applied to the quadriceps muscles of the participant to elicit a movement and the knee joint angle is measured using an encoder. The amplitude of the of the stimulation was selected such that for each participant the joint angle was greater than $10^{\circ}$, but less than $70^{\circ}$. This ensured that the muscles were always in tension and sufficiently far from hyperextension/hyperflexion. The parameters estimated in Tests 1-4, were used to populate the model of the knee extension, and then an optimization was performed to identify the force-velocity parameter $\left(c_{3}\right.$ in (3)) that makes the model best match the measured data. Fig. 8 compares the measured knee joint angle to the knee joint angle of the model when given the same input. The output of the model matches the measured joint angle with an RMS error of $3.15^{\circ}$, which illustrates that the model and the estimated parameters are a close match to the actual system. Errors in the parameter estimation process could have resulted from unmodeled musculoskeletal dynamics, such as the electromechanical delay [50] or muscle fatigue dynamics [40].

The results of the parameter estimation for all three participants are shown in Table 1. The parameters in Table 1 are subject specific, and can vary depending on the stimulation train that is used. The RMS errors of the fits resulting from the push/pull, isometric contraction, muscle activation, pendulum, and sinusoidal input tests are given in Table 1. 


\section{Value}

\begin{tabular}{|c|c|c|c|}
\hline Parameter & Participant 1 & Participant 2 & Participant 3 \\
\hline$\alpha\left[\frac{1}{\mathrm{~kg} \mathrm{~m}^{2}}\right]$ & 1.29 & 1.17 & 1.31 \\
\hline$\beta\left[\frac{\mathrm{N}}{\mathrm{kg} \mathrm{m}^{2}}\right]$ & 40.3 & 42.4 & 40.9 \\
\hline$\phi_{0}$ [radians] & $2.81 \times 10^{-14}$ & $9.10 \times 10^{-4}$ & $3.78 \times 10^{-6}$ \\
\hline$d_{1}[\mathrm{Nm}]$ & 0.863 & 9.03 & 6.99 \\
\hline$d_{2}[\mathrm{Nms}]$ & 2.56 & 5.52 & 3.52 \\
\hline$d_{3}[\mathrm{Nm}]$ & $1 \times 10^{-10}$ & $1 \times 10^{-12}$ & $1.19 \times 10^{-11}$ \\
\hline$d_{4}$ & 15.9 & 16.0 & 15.9 \\
\hline$d_{5}[\mathrm{Nm}]$ & 0.405 & 3.68 & 0.0302 \\
\hline$d_{6}$ & -94.2 & -1.72 & -45.1 \\
\hline$c_{0}[\mathrm{Nm}]$ & 50.9 & 58.1 & 41.9 \\
\hline$c_{1}[\mathrm{Nm}]$ & 163 & 68.3 & 21.1 \\
\hline$c_{2}[\mathrm{Nm}]$ & -125 & -47.9 & -23.0 \\
\hline$c_{3}$ & 1.28 & 0.990 & 0.0400 \\
\hline$\theta_{e q}[$ radians] & 0.416 & 0.309 & 0.264 \\
\hline$T_{a}$ [seconds] & 0.18 & 0.24 & 0.2 \\
\hline$I_{t}[\mathrm{~mA}]$ & 23.2 & 26.0 & 39.0 \\
\hline$I_{s}[\mathrm{~mA}]$ & 76.8 & 79.0 & 80 \\
\hline Test 2 Fit RMS[Nm] & 0.264 & 0.928 & 0.352 \\
\hline Test 3 Isometric Fit RMS [Nm] & 7.97 & 2.352 & 8.53 \\
\hline Test 3 Activation Fit RMS & 0.0373 & 0.0397 & 0.0431 \\
\hline Test 4 RMS Fit [deg.] & 7.78 & 8.68 & 7.72 \\
\hline Test 5 RMS Fit [deg.] & 3.15 & 7.94 & 7.47 \\
\hline
\end{tabular}

Table 1: Estimated parameters of each able-bodied participant. These parameters are subject specific, and can change with the stimulation pulse train used. For the parameter estimation and MPC experiments $400 \mu \mathrm{s}$ bi-phasic rectangular pulse trains at $35 \mathrm{~Hz}$ were used. 


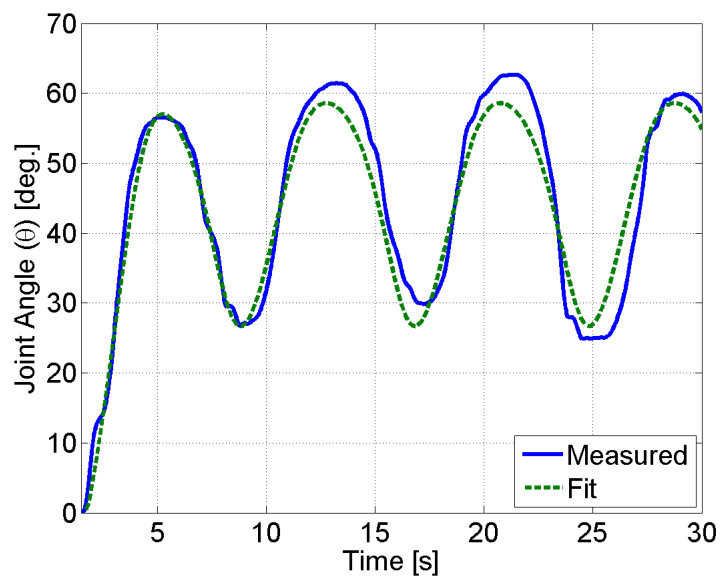

Figure 8: A known, sinusoidal stimulation was applied to the quadriceps and the resulting joint angle was measured. This plot shows the measured joint angle and the joint angle predicted by the model with the estimated parameters.

\section{Gradient Projection Model Predictive Control}

A NMPC algorithm was used to achieve greater accuracy in the control, because the FES leg extension system is nonlinear. Because the system dynamics are continuously differentiable, a gradient projection algorithm may be used. This will provide sufficiently fast computation times to allow real time implementation of the MPC [19] and [25]. Gradient projection MPC, or GRAMPC $[25,26]$, uses the gradient of the Hamiltonian and a projection function to solve the optimal control problem. The gradient projection algorithm also uses an early termination condition to ensure that the algorithm can be implemented in real time. This section will define the optimal control problem and give a brief summary of the gradient projection algorithm that was used to solve the optimal control problem.

The optimal control problem is stated as

$$
\begin{array}{rc}
\min _{u} & J(x, u)=V(x(T))+\int_{t_{0}}^{T} l(x(\tau), u(\tau)) d \tau \\
\text { subject to: } & \dot{x}(t)=f(x, u) \\
& x\left(t_{0}\right)=x_{0} \\
u \in \mathcal{U}
\end{array}
$$

where $x_{0} \in \mathbb{R}^{3}$ is the initial condition of the states at each time step, $\mathcal{U}=$ $\left[u^{-}, u^{+}\right]$are the bounds on $u, t_{0}$ is the initial time of the horizon, and $T$ is the final time of the horizon. The objective of the optimal control problem is to solve for the control trajectory, $u$, that minimize the cost function $J(x, u) \in \mathbb{R}^{+}$over a finite time horizon $t \in\left[t_{0}, T\right]$ while satisfying the constraints. For this study, 
the only control input to the system is the normalized stimulation. Therefore, $u=u_{k e} \in[0,1]$ which means that $u^{-}=0$ and $u^{+}=1$.

Let the integral cost function, $l(x, u)$, and the terminal cost, $V(x(T))$, in $(7)$ be quadratic functions defined as

$$
\begin{gathered}
l(x, u)=\Delta x^{T} Q \Delta x+\Delta u^{T} R \Delta u \\
V(x(T))=\Delta x(T)^{T} P \Delta x(T)
\end{gathered}
$$

where $\Delta x=x-x_{\text {des }}, \Delta u=u-u_{\text {des }}, x_{\text {des }} \in \mathbb{R}^{3}$ is the desired state, and $u_{\text {des }} \in \mathcal{U}$ is the desired control trajectory. The weight matrices $Q \in \mathbb{R}^{3 \times 3}, R \in \mathbb{R}$, and $P \in \mathbb{R}^{3 \times 3}$ must be at least positive semi-definite and can be tuned to achieve the desired performance.

Pontryagin's Minimum Principle states that $u^{*}(t)$ for $t \in[0, T)$ solves the optimal control problem if it minimizes the Hamiltonian [41], where the Hamiltonian is defined as

$$
H(x, \lambda, u)=l(x, u)+\lambda^{T} f(x, u),
$$

where $\lambda(t) \in \mathbb{R}^{n}$ is the costate vector. Therefore, the gradient of the Hamiltonian can be used to iteratively solve for the optimal control trajectory, $u^{*}(t)$. During the iterative solution process the gradient projection algorithm uses a projection function, defined as

$$
\psi(u)= \begin{cases}u^{-} & u<u^{-} \\ u, & u^{-} \leq u \leq u^{+} \\ u^{+} & u^{+}<u\end{cases}
$$

which ensures that all values in the control trajectory remain in the set $\mathcal{U}$.

The detailed computation steps of the gradient projection algorithm can be found in [19], but can be summarized as follows for each discrete time step $k$ :

1. Initialization: $j=0$

(a) Set the convergence tolerance $\varepsilon_{j}$.

(b) Choose initial control trajectory $u_{k}^{(0)} \in \mathcal{U}_{[0, T]}$.

(c) Integrate the system dynamics forward in time to solve for $x_{k}^{(0)}$ given $u_{k}^{(0)}$.

2. Gradient Step: While

$\left|J\left(x_{k}^{(j+1)}, u_{k}^{(j+1)}\right)-J\left(x_{k}^{(j)}, u_{k}^{(j)}\right)\right|>\varepsilon_{j}$ or $j \leq N$

(a) Integrate $\dot{\lambda}=-\left.\frac{\partial H(x, \lambda, u)}{\partial x}\right|_{x=x_{k}^{(j)}, u=u_{k}^{(j)}}$ backward in time to solve for the costates, where $\lambda_{k}^{(j)}(T)=\frac{\partial V\left(x_{k}^{(j)}(T)\right)}{\partial x(T)}$ is the terminal condition.

(b) Compute the search direction, $s_{k}^{(j)} \in \mathbb{R}$, from the Hamiltonian as $s_{k}^{(j)}=-\left.\frac{\partial H(x, \lambda, u)}{\partial u}\right|_{x=x_{k}^{(j)}, u=u_{k}^{(j)}, \lambda_{k}^{(j)}}$. 
(c) Compute the step size, $\alpha_{k}^{(j)} \in \mathbb{R}^{+}$, by solving the line search problem $\alpha_{k}^{(j)}=\underset{\alpha>0}{\operatorname{argmin}} J\left(x_{k}^{(j)}, \psi\left(u_{k}^{(j)}+\alpha s_{k}^{(j)}\right)\right.$ ) (this can be approximately solved to decrease computation time using a number of methods [19]).

(d) Compute the new control trajectory $u_{k}^{(j+1)}=\psi\left(u_{k}^{(j)}+\alpha_{k}^{(j)} s_{k}^{(j)}\right)$, where $\psi(\cdot)$ is the projection function in (10).

(e) Integrate the system dynamics forward in time to solve for $x_{k}^{(j+1)}$ given $u_{k}^{(j+1)}$ and evaluate the cost function $J\left(x_{k}^{(j+1)}, u_{k}^{(j+1)}\right)$.

(f) Quit if $\left|J\left(x_{k}^{(j+1)}, u_{k}^{(j+1)}\right)-J\left(x_{k}^{(j)}, u_{k}^{(j)}\right)\right| \leq \varepsilon_{j}$ or if $j$ has exceeded the max iteration limit, $N$. Otherwise set $j=j+1$ and reiterate gradient step.

When the iterative loop terminates due to the change in the cost being within the convergence tolerance the resulting control trajectory is said to be optimal. However, when the iterative loop terminates early (terminates because $j$ is greater than the max iteration limit specified by the user) the resulting control trajectory is said to be suboptimal. To increase the convergence rate of this algorithm, and improve upon previously computed suboptimal solutions, the solution from the previous time step is used to initialize the control trajectory at the next time step. In other words, if $u_{k}^{*}$ is the computed control trajectory at the $k^{\text {th }}$ time step then at the next time step $u_{k+1}^{(0)}=u_{k}^{*}$ is used as the initial condition. This warm-start technique improves the convergence rate at each time step and can help guide suboptimal solutions to eventually converge to the optimal solution.

Some MPC algorithms use a terminal constraint in the definition of the optimal control problem to ensure stability of the MPC [6]. However, instead of using a terminal constraint to stabilize the MPC, a control Lyapunov function can be used as the terminal cost, $V(x(T))$, to ensure the stability of the MPC for the optimal and suboptimal cases, given that the set $\left(x_{d e s}, u_{d e s}\right)$ is an equilibrium point of $f(x, u)$. For the dynamic system presented in this paper, given a desired joint angle the remaining elements of $x_{\text {des }}$ and $u_{\text {des }}$ can be solved from (6) as $f\left(x_{d e s}, u_{d e s}\right)=0$. For the leg extension neuroprosthesis system presented in this paper given a desired joint angle the remaining elements of $x_{d e s}$ and $u_{d e s}$ can be solved from $(6)$ as $f\left(x_{\text {des }}, u_{\text {des }}\right)=0$. The control Lyapunov condition is approximately satisfied by solving the algebraic Riccati equation [19, 20]

$$
P A+A^{T} P-P B R^{-1} B^{T} P+Q=0
$$

for the gain matrix $P$, where the matrices $A$ and $B$ are the matrices of the linearized state-space system that can be computed using Jacobian linearization 
about the desired states and control signals ( $x_{\text {des }}$ and $u_{\text {des }}$, respectively) as

$$
\begin{aligned}
A & =\left.\frac{\partial f(x, u)}{\partial x}\right|_{x=x_{\text {des }}, u=u_{\text {des }}} \\
& =\left[\begin{array}{ccc}
0 & 1 & 0 \\
A_{21} & A_{22} & A_{23} \\
0 & 0 & -\frac{1}{T_{a}}
\end{array}\right] \\
B & =\left.\frac{\partial f(x, u)}{\partial u}\right|_{x=x_{\text {des }}, u=u_{\text {des }}} \\
& =\left[\begin{array}{c}
0 \\
0 \\
\frac{1}{T_{a}}
\end{array}\right]
\end{aligned}
$$

where

$$
\begin{aligned}
A_{21}= & -\beta \cos \left(x_{d e s}^{(1)}+\theta_{e q}\right) \\
- & \alpha\left[d_{1}+d_{3} d_{4} \mathrm{e}^{d_{4}\left(\frac{\pi}{2}-x_{d e s}^{(1)}-\theta_{e q}\right)}\right. \\
- & d_{5} d_{6} \mathrm{e}^{d_{6}\left(\frac{\pi}{2}-x_{d e s}^{(1)}-\theta_{e q}\right)} \\
+ & \left(2 c_{2}\left(\frac{\pi}{2}-x_{d e s}^{(1)}-\theta_{e q}\right)\right. \\
& \left.\left.+c_{1}\right)\left(1-c_{3} x_{d e s}^{(2)}\right) x_{d e s}^{(3)}\right] \\
A_{22}=-\alpha & {\left[d_{2}+\left(c_{2}\left(\frac{\pi}{2}-x_{d e s}^{(1)}-\theta_{e q}\right)^{2}\right.\right.} \\
+ & \left.\left.c_{1}\left(\frac{\pi}{2}-x_{d e s}^{(1)}-\theta_{e q}\right)+c_{0}\right) c_{3} x_{d e s}^{(3)}\right], \\
A_{23}= & \alpha\left[\left(c_{2}\left(\frac{\pi}{2}-x_{d e s}^{(1)}-\theta_{e q}\right)^{2}\right.\right. \\
& +c_{1}\left(\frac{\pi}{2}-x_{d e s}^{(1)}-\theta_{e q}\right) \\
& \left.\left.+c_{0}\right)\left(1-c_{3} x_{d e s}^{(2)}\right)\right]
\end{aligned}
$$

and $x_{\text {des }}^{(j)}$ for $j=\{1,2,3\}$ is the $j$ th element of $x_{d e s}$.

\section{Results}

The gradient projection algorithm NMPC was tested in simulations and in experiments on three able-bodied male participants between the ages of 25-28 years $^{2}$. The participants were seated in a leg extension machine, facing away

\footnotetext{
${ }^{2}$ Prior to any experimentation, approval from the Institutional Review Board at the University of Pittsburgh was obtained.
} 
from the computer screen so that they could not see the desired or measured positions. The leg extension machine was fitted with a CALT GHH100 rotary encoder (Shanghai Qiyi Electrical \& Mechanical Equipment Co. Ltd) mounted to the arm of the leg extension machine to measure the joint angle. Position is the only state that is measured, the velocity and activation states are estimated using different methods. The angular velocity is estimated from the joint angle using a discrete time derivative, while the muscle activation is estimated using the normalized stimulation and the model of muscle activation that was previously estimated. The participants were asked to relax and avoid any voluntary contractions during the experiments. The simulation results are plotted with the experimental results to compare the performance of the controller with exact model knowledge (simulations) against the performance of the controller on the real system (experiments). A sampling frequency of $100 \mathrm{~Hz}$ was used for the control, the horizon of the NMPC was set at 0.5 second, and an adaptive line search method was used to compute the step size. The sampling frequency of the control $(100 \mathrm{~Hz})$ is approximately three times faster than the stimulation frequency $(35 \mathrm{~Hz})$. Therefore, it was assumed that the sampling frequency of the control system was sufficiently fast, such that it does not affect the stability of the system. However, The NMPC used Heun's method, a second order numerical integration, to solve the dynamics function and the Trapezoidal rule was used to integrate the definite integral of the cost function. Through simulations the order of these numerical methods were found to be sufficient in approximating the continuous time optimal control problem. The NMPC was implemented in Simulink (MathWorks, Inc.) using a QPIDe data acquisition board (Quanser, Inc.) to collect data from the encoder, and an FNS-16 Multi-Channel Stimulator (CWE, Inc.) to generate the electrical stimulation.

The desired states and input, used in simulations and experiments, were defined as

$$
x_{\text {des }}=\left\{\begin{array}{l}
{\left[\begin{array}{l}
0 \\
0 \\
0
\end{array}\right], \quad t \leq 5} \\
{\left[\begin{array}{c}
40^{\underline{0}} \\
0 \\
a_{e q}
\end{array}\right], \quad t>5}
\end{array} \quad u_{\text {des }}=\left\{\begin{array}{ll}
0, & t \leq 5 \\
u_{e q}, & t>5
\end{array},\right.\right.
$$

where $a_{e q}$ and $u_{e q}$ are the muscle activation and normalized stimulation amplitude that make $40^{\circ}$ the equilibrium position. These values are determined by solving for the equilibrium of the dynamics (see (6)) for the given desired knee angle of $40^{\circ}$. These values are subject specific and can be determined using the estimated parameters for each of the test participants.

The weight matrices in the integral cost function were tuned to achieve good steady-state performance for each participant. As an example of appropriate cost weight matrices the values that were used in experiments and simulations 


\begin{tabular}{cccc}
\hline Participant \# & 1 & 2 & 3 \\
\hline Transient RMS Error [deg.] & 14.4 & 13.5 & 13.4 \\
\hline SSRMS Error [deg.] & 0.841 & 1.45 & 1.71 \\
\hline
\end{tabular}

Table 2: Transient (5-10s) and steady-state (10-30s) RMS errors from experimental trials of the NMPC for all three participants.

for Participant 1 are

$$
Q=\left[\begin{array}{ccc}
10 & 0 & 0 \\
0 & 5 & 0 \\
0 & 0 & 1
\end{array}\right] \text { and } R=1
$$

Because $Q$ is a diagonal matrix with all positive elements and $R$ is a positive scalar it is clear that both $Q$ and $R$ are positive definite. The algebraic Riccati equation was solved using the weight matrices $Q$ and $R$, and the linearized matrices $A$ and $B$ that resulted from using the subject specific parameters for Participant 1

$$
A=\left[\begin{array}{ccc}
0 & 1 & 0 \\
-41.95 & -32.04 & -121.46 \\
0 & 0 & -6.25
\end{array}\right] \text { and } B=\left[\begin{array}{c}
0 \\
0 \\
6.25
\end{array}\right] \text {. }
$$

The resulting weight matrix of the terminal cost for Participant 1 was computed to be

$$
P=\left[\begin{array}{ccc}
7.22 & 0.102 & -0.193 \\
0.102 & 0.0710 & -0.129 \\
-0.193 & -0.129 & 0.765
\end{array}\right]
$$

Although it is not apparent, because $P$ is a Hermitian matrix with all positive eigenvalues it can be shown that the matrix is positive definite. Since $Q, R$, and $P$ are all positive definite it can be concluded that the quadratic cost function is strictly positive.

The experimental and simulation results of the NMPC for regulation at a knee angle of $40^{\circ}$ are shown in Fig. 9, and the steady-state root mean square (SSRMS) (10-30 seconds) errors are given in Table 2. One notable difference is oscillations that occur shortly after the onset of the step, which is most likely a result of errors in the estimation of the parameters or unmodeled dynamics.

Although the terminal cost gain matrix that stabilizes the system is specific to a knee angle of $40^{\circ}$, regulation of the knee angle to different reference positions was also tested through simulations and experiments. Fig. 10 shows the results of simulations and experiments when the reference position is varied. The controller was given a reference of $40^{\circ}$ for ten seconds, followed by a reference of $20^{\circ}$ for ten seconds, and then a reference of $60^{\circ}$ for ten seconds. It took approximately five seconds for the transient response to decay, so SSRMS errors 

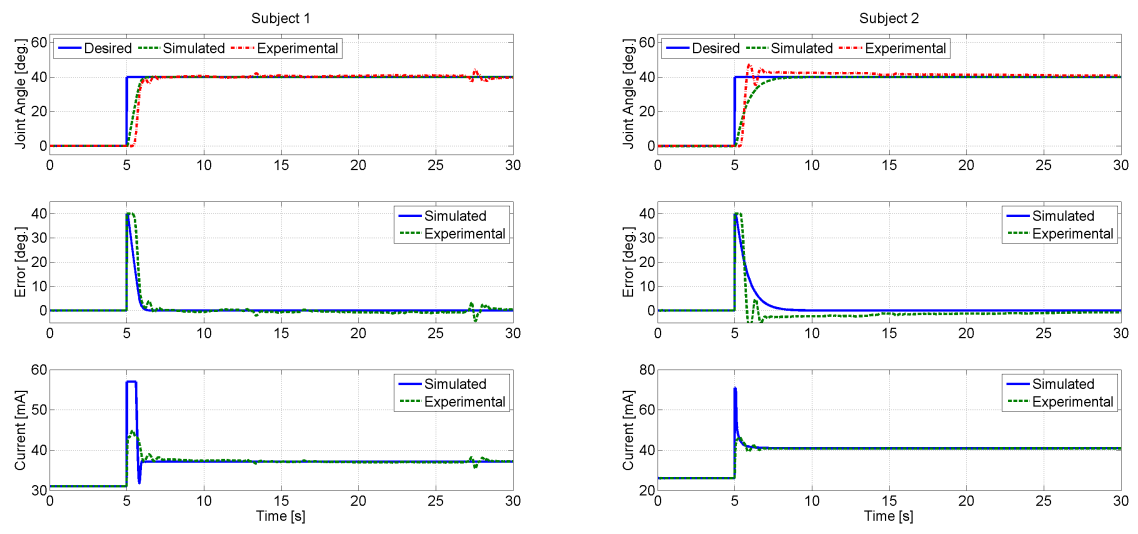

(a)

(b)
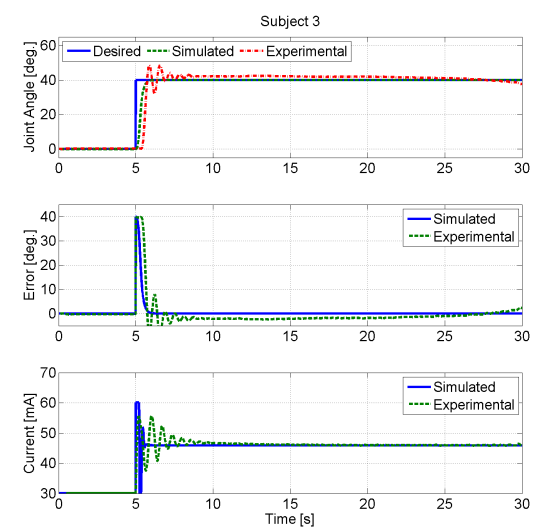

(c)

Figure 9: Results of NMPC of FES on three able-bodied participants. The experimental results were plotted with the simulation results, both using the same cost functions and subject parameters, to illustrate how the controller would perform in the ideal case of exact model knowledge. 


\begin{tabular}{cccc}
\hline Participant \# & 1 & 2 & 3 \\
\hline Transient RMS Error [deg.] (5-10s) & 15.0 & 13.0 & 13.6 \\
\hline SSRMS Error [deg.] (10-15s) & 1.15 & 0.920 & 1.00 \\
\hline Transient RMS Error [deg.] (15-20s) & 10.8 & 8.40 & 6.72 \\
\hline SSRMS Error [deg.] (20-25s) & 2.34 & 1.89 & 2.70 \\
\hline Transient RMS Error [deg.] (25-30s) & 15.0 & 12.7 & 13.6 \\
\hline SSRMS Error [deg.] (30-35s) & 3.44 & 2.51 & 1.38 \\
\hline
\end{tabular}

Table 3: Transient and steady-state (SS) RMS errors calculated for the knee regulation experiments with step changes.

\begin{tabular}{cccc}
\hline Participant \# & 1 & 2 & 3 \\
\hline Transient RMS Error [deg.] & 17.07 & 13.2 & 13.9 \\
\hline SSRMS Error [deg.] & 1.12 & 1.03 & 2.05 \\
\hline
\end{tabular}

Table 4: Transient (5-10 seconds) and steady-state(SS) RMS (10-30 seconds) errors from experimental trials of the NMPC with impulse disturbances with a magnitude of approximately $10 \mathrm{Nm}$ at approximately 15 and 25 seconds during the experiments.

were calculated using the last five seconds that the lower limb was regulated at each of the three reference positions. This corresponds to experiment times of 10-15 seconds for $40^{\circ}, 20-25$ seconds at $20^{\circ}$, and $30-35$ seconds at $60^{\circ}$. The SSRMS errors for these times are given in Table 3.

To determine the robustness of the NMPC to disturbances an impulse was applied during regulation experiments for each of the three participants. This was accomplished by tapping the arm of the leg extension machine during the steady-state response of the regulation experiment. The results of these experiments for all three participants are shown in Fig. 11. The individual conducting the experiments applied the disturbances at approximately 15 and 25 seconds during the experiments, and the disturbance torque was measured using the load cell that attaches the leg to the arm of the leg extension machine. It can be observed from Fig. 11 that the disturbances had a magnitude of approximately $10 \mathrm{Nm}$, and the measured joint angles deviated only slightly from the desired joint angle at the instants that the disturbances were applied. The steady-state RMS errors during these experiments for all three participants are given in Table 4.

To determine if NMPC is causing less muscle fatigue than typical feedback controllers by allocating the optimal stimulation, regulation experiments were performed with the NMPC and a PID controller on separate days. Since muscle 

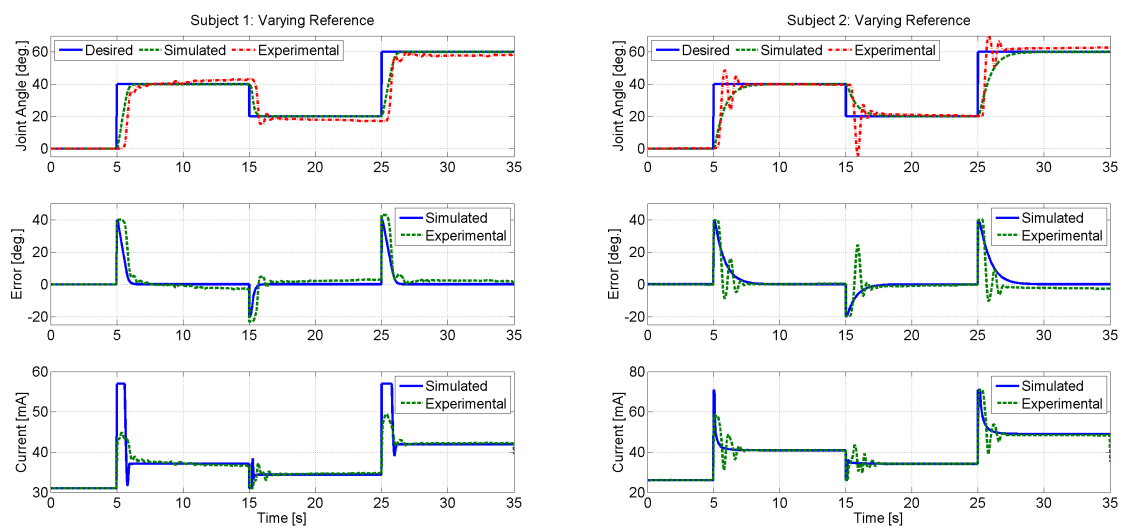

(a)

(b)
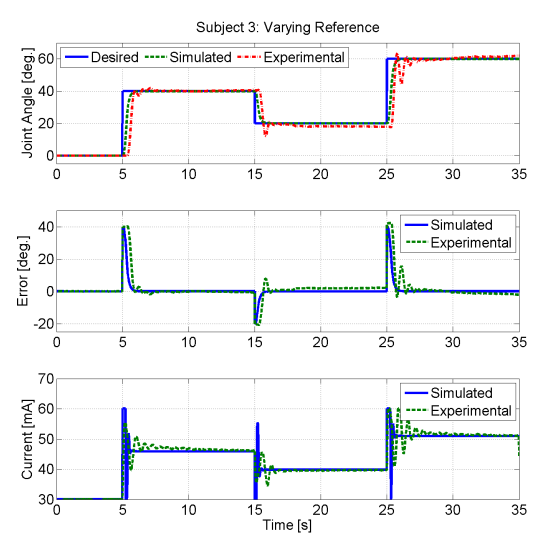

(c)

Figure 10: Simulations and experiments of the NMPC regulating the knee angle to different reference positions for three participants. The controller was given a reference of $40^{\circ}$ for $5-15$ seconds, followed by a reference of $20^{\circ}$ for $15-25$ seconds, and then a reference of $60^{\circ}$ for $25-35$ seconds. 

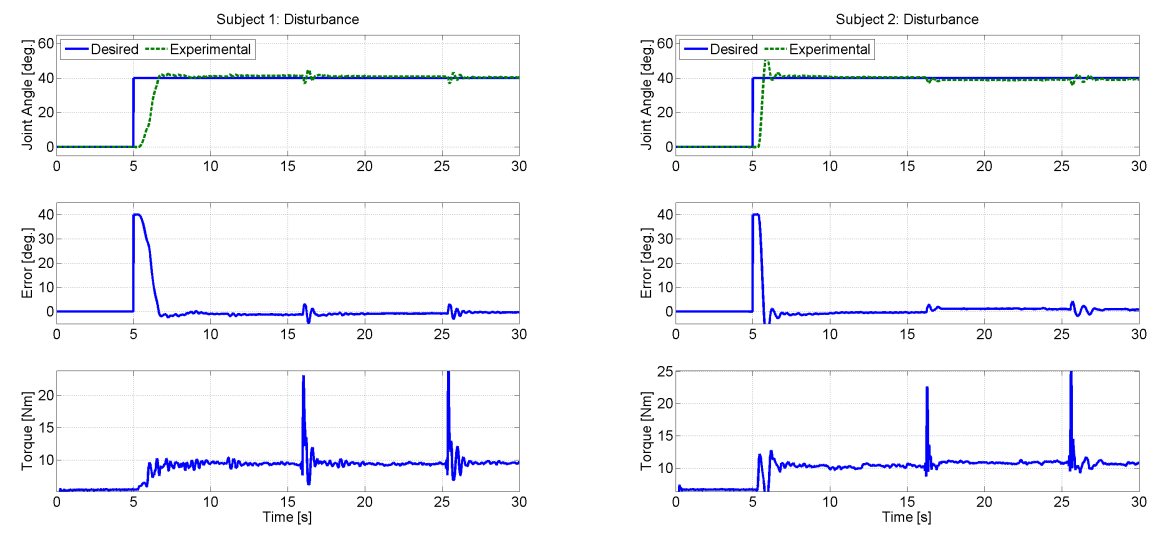

(a)

(b)
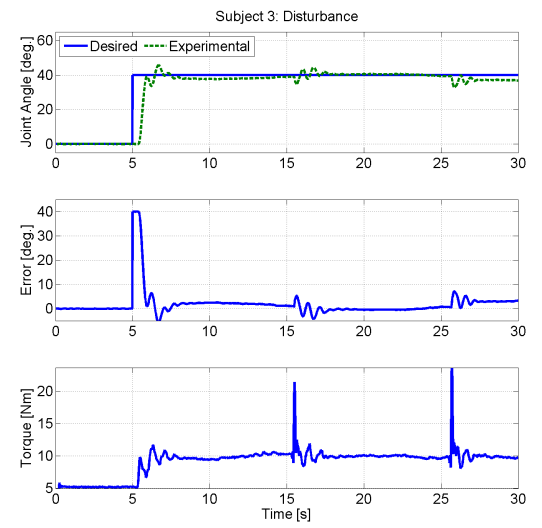

(c)

Figure 11: Results from disturbance rejection experiments. Impulse disturbances were applied by tapping the arm of the leg extension machine at approximately 15 and 25 seconds during a regulation experiment. 
fatigue is a decrease in the force that the muscle can produce, isometric contraction tests were used immediately after each regulation experiment (NMPC and PID) as a measurement of muscle fatigue. To ensure that muscle fatigue occurred during the regulation experiments the experiments were conducted for one minute and $15 \mathrm{lbs}$ were added to the arm of the leg extension machine. This added weight was compensated for in the NMPC by adjusting the mass and inertia parameters in the model. Immediately after each regulation experiment a 30 second long isometric contraction test was used to determine how much each controller fatigued the muscle. Since there can be some variation in muscle force a brief isometric contraction test was used before each regulation experiment, and the peak torque measured was used to normalize the isometric contraction tests that were performed after the regulation experiments. The initial normalized isometric contraction, regulation experiment (NMPC and PID), and the final normalized isometric contraction for one of the participants are shown in Fig. 12.

The peak torque values of the isometric contractions before the regulation experiments were $69.6 \mathrm{Nm}$ and $73.9 \mathrm{Nm}$ for the NMPC and PID, respectively. The steady-state RMS values from the regulation experiments, shown in Fig. 12(b) , were computed to be $3.70^{\circ}$ for the PID controller and $6.17^{\circ}$ for the NMPC. The RMS values of the current were computed to be $51 \mathrm{~mA}$ for the NMPC and $55 \mathrm{~mA}$ for the PID controller. This illustrates that the PID controller was able to achieve better performance than the NMPC controller by increasing the current amplitude as fatigue occurred. Immediately after each regulation experiment an isometric contraction test was used to measure how much the muscles had fatigued. These are shown in Fig. 12(c). Since variation in muscle force can occur, the isometric contraction test was conducted for 30 s and the integral of the normalized torque measurements were used as a metric for how much the muscles had fatigued. The normalized torque integral for the NMPC was computed to be $19.1 \mathrm{~s}$, and the normalized torque integral for the PID was computed to be $14.2 \mathrm{~s}$. This demonstrates that the muscles were more fatigued after the PID regulation experiment than after the NMPC regulation experiment.

\section{Discussion}

A real time implementation of a NMPC algorithm seems promising for its use in FES control systems. The experimental results illustrate that the NMPC can be implemented despite external disturbances and uncertainties in the identified muscle model. The settings of the NMPC algorithm that were used in the experiments resulted in an average computation time of $2.0 \mathrm{~ms}$ at each time step, which is clearly faster than the $10 \mathrm{~ms}$ sampling period used in the control. Further modifications to the settings of the NMPC algorithm may be made to decrease the computation time further; however, the settings that were used here were chosen because they yielded a sufficient computational precision and solve time. The muscle model for the NMPC algorithm that is used in this paper is

a Hill-type muscle model. However, the NMPC control technique may be used 


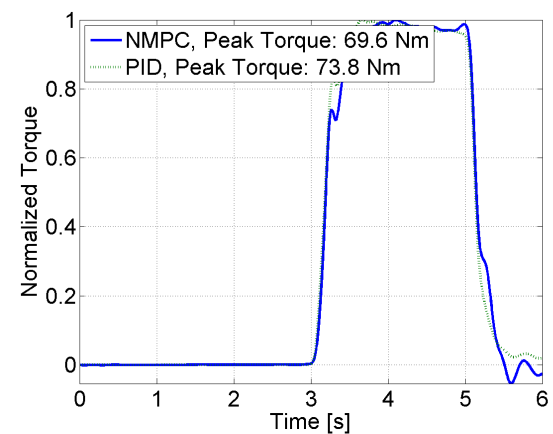

(a)
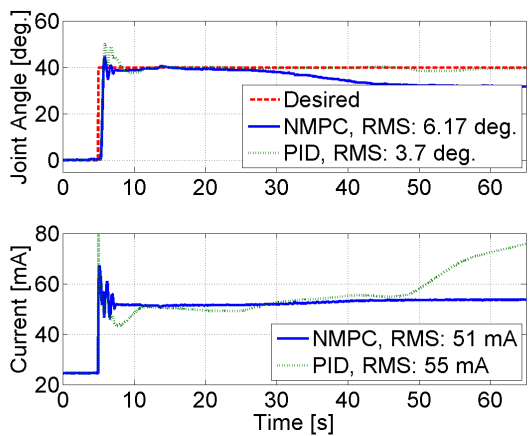

(b)

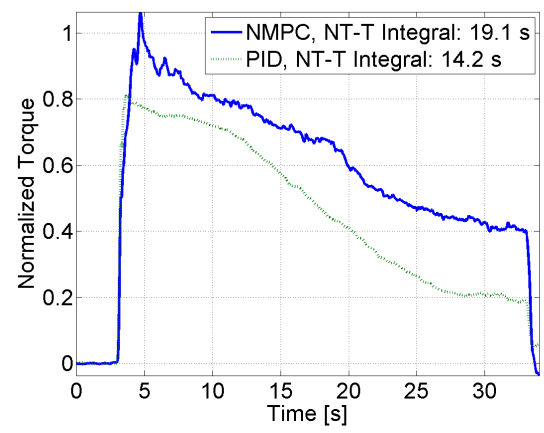

(c)

Figure 12: Regulation tests were performed with the NMPC controller and a PID controller. The NMPC test and PID test were performed on separate days so that muscle fatigue that occurs during one test does not affect the other test. Since muscle force can vary from dayto-day first an isometric contraction test is performed (a) so that the load cell measurement can be normalized. Then a 60 second long regulation test was performed (b). To determine how fatigued the leg was after the regulation experiment another isometric contraction test was performed (c). The results of the second isomeric contraction tests are normalized and then the integral of the normalized torques were computed. A lower normalized torque-time (NT-T) integral indicates more fatigue. 
with other muscle models that may have a simpler identification process or yield a more accurate model of the nonlinear musculoskeletal dynamics. For example, other phenomenological muscle models such as a Hill/Huxley type model may be used [10-12]. Non-phenomenological models, such as Hammerstein models [18, 32], Sigmoid functions [43], and neural networks [7], have also been used to model the muscles response to FES and may be extended to an MPC framework.

Using the models developed in $[14,37,45]$ this work can be extended to a complete gait system that includes models of hip, knee, and ankle flexors/extensors. The model developed in this paper is not immediately valid for walking; however, many of the elements identified in this model may remain valid. For example, the methods used to identify shank mass/inertial parameters, joint torque-length, and joint torque-velocity relationships can be similarly used to identify these relationships for other limb joints. In a recent work by Farris et al. [16], a PD/PID control was used for tracking control of limb joints in a finite state machine (FSM) control of a powered walking exoskeleton. The MPC technique can be used for a single joint control, instead of PD/PID control, in a finite state machine control for producing walking movements.

Although further experiments with a larger population size are necessary, these preliminary results indicated that the NMPC may cause less muscle fatigue. However, this comes at the cost of performance. In the presented results the isometric contraction after the NMPC experiment had a normalized forcetime integral of 19.1s, while the isometric contraction after the PID experiment had a normalized force-time integral of 14.2s. This indicates that the muscle was more fatigued after the PID regulation experiment than the NMPC regulation experiment. However, although the force-time integral of the NMPC was higher there was a noticeable drop in performance after 30 seconds in the regulation experiment while the performance of the PID controller remained approximately the same. The steady-state RMS error for the NMPC was computed to be $6.17^{\circ}$, while the stead-state RMS error of the PID controller was computed to be $3.70^{\circ}$. These results were somewhat expected, since the NMPC controller is minimizing a cost function that contains both performance (minimize error) and effort (minimize control). In this case performance and effort are conflicting terms (reducing error requires more effort), and the NMPC solves for the control effort that achieves balance between the two that minimizes the overall cost function. As previously stated this experiment will need to be conducted on a larger population size to determine if the differences observed in these preliminary results are statistically significant.

\section{Conclusion}

A gradient projection based NMPC was presented for regulating a limb joint angle through FES. The NMPC was validated through knee extension experiments on 3 able-bodied persons. A procedure for estimating parameters of a musculoskeletal model, which were used to implement NMPC, was also presented. The experimental results show that the NMPC is capable of controlling FES to regulate a joint angle with a steady-state RMS error of approximately 
$2^{\mathrm{o}}$, even in the presence of a disturbance. The results illustrate that the gradient projection based NMPC can be implemented in real time with a sampling frequency of $100 \mathrm{~Hz}$ for joint angle regulation of FES-based devices. Future work will explore robustifying techniques for MPC such as tube-based MPC [34]. These techniques will make the MPC more robust to unmodeled dynamics, such as electromechanical delay present in musculoskeletal systems [2, 44, 50] and other modeling uncertainties. The results of this research will be used to inform the control of a hybrid (FES and electric motor) walking exoskeleton being developed in our laboratory[28]. Potentially, the NMPC algorithm can be used to determine the minimum amount of stimulation and motor torque necessary to create the gait motion in existing hybrid walking exoskeletons [9, 17], and [22].

\section{Acknowledgments}

This work was funded in part by the National Science Foundation award number 1462876 and 1511139, and the University of Pittsburgh's Competitive Medical Research Fund and Central Research Development Fund.

\section{References}

[1] A. Ajoudani and A. Erfanian. A neuro-sliding-mode control with adaptive modeling of uncertainty for control of movement in paralyzed limbs using functional electrical stimulation. IEEE Transactions on Biomedical Engineering, 56(7):1771-1780, 2009.

[2] N. Alibeji, N. Kirsch, S. Farrokhi, and N. Sharma. Further results on predictor-based control of neuromuscular electrical stimulation. IEEE Trans. Neural Syst. Rehabil. Eng., Pre-print available online.

[3] T. Bajd, A. Kralj, R. Turk, H. Benko, and J. Šega. The use of a fourchannel electrical stimulator as an ambulatory aid for paraplegic patients. Phys. Ther., 63:1116-1120, 1983.

[4] M. Benoussaad, K. Mombaur, and C. Azevedo-Coste. Nonlinear model predictive control of joint ankle by electrical stimulation for drop foot correction. In IEEE/RSJ Int. Conf. on IROS, pages 983-989. IEEE, 2013.

[5] C. Bickel, C. Gregory, and J. Dean. Motor unit recruitment during neuromuscular electrical stimulation: a critical appraisal. European Journal of Applied Physiology, 111(10):2399-2407, 2011.

[6] H. Chen and F. Allgöwer. A quasi-infinite horizon nonlinear model predictive control scheme with guaranteed stability. Automatica, 34(10):1205$1217,1998$. 
[7] T. Cheng, Q. Wang, R. Kamalapurkar, H. Dinh, M. Bellman, and W. Dixon. Identification-based closed-loop NMES limb tracking with amplitude-modulated control input. IEEE Trans. Cybern. (Accepted), 2015.

[8] A. del Ama, Á. Gil-Agudo, E. Bravo-Esteban, S. Pérez-Nombela, J. Pons, and J. Moreno. Hybrid therapy of walking with kinesis overground robot for persons with incomplete spinal cord injury: A feasibility study. Rob. Auton. Syst., 2014.

[9] A. del Ama, Á. Gil-Agudo, J. Pons, and J. Moreno. Hybrid FES-robot cooperative control of ambulatory gait rehabilitation exoskeleton. J. Neuroeng. Rehabil., 11(1):27, 2014.

[10] J. Ding, A. Wexler, and S. Binder-Macleod. A predictive fatigue model. I. predicting the effect of stimulation frequency and pattern on fatigue. IEEE Trans. Rehabil. Eng., 10(1):48-58, 2002.

[11] J. Ding, A. Wexler, and S. Binder-Macleod. A predictive fatigue model. II. predicting the effect of resting times on fatigue. IEEE Trans. Rehabil. Eng., 10(1):59-67, 2002.

[12] B. Doll, N. Kirsch, and N. Sharma. Optimization of a stimulation train based on a predictive model of muscle force and fatigue. In Proceedings of 9th IFAC Symposium on Biological and Medical Systems, Berlin, Germany (Accepted), 2015.

[13] A. Dollar and H. Herr. Lower extremity exoskeletons and active orthoses: challenges and state-of-the-art. IEEE Transactions on Robotics, 24(1):144$158,2008$.

[14] S. Dosen and D. Popovic. Moving-window dynamic optimization: design of stimulation profiles for walking. IEEE Trans. Biomed. Eng., 56(5):12981309, 2009.

[15] R. Esfanjani and F. Towhidkhah. Application of nonlinear model predictive controller for FES-assisted standing up in paraplegia. In IEEE Eng. Med. Biol. Soc., volume 6, pages 6210-6213, 2005.

[16] R. Farris, H. Quintero, and M. Goldfarb. Preliminary evaluation of a powered lower limb orthosis to aid walking in paraplegic individuals. IEEE Trans. Neural Syst. Rehabil. Eng., 19(6):652-659, 2011.

[17] R. Farris, H. Quintero, S. Murray, K. Ha, C. Hartigan, and M. Goldfarb. A preliminary assessment of legged mobility provided by a lower limb exoskeleton for persons with paraplegia. IEEE Transactions on Neural Systems and Rehabilitation Engineering, 22(3):482-490, May 2014.

[18] C. Freeman, A. Hughes, J. Burridge, P. Chappell, P. Lewin, and E. Rogers. A model of the upper extremity using FES for stroke rehabilitation. Journal of biomechanical engineering, 131(3):031011, 2009. 
[19] K. Graichen and B. Käpernick. A real-time gradient method for nonlinear model predictive control. INTECH Open Access Publisher, 2012.

[20] K. Graichen and A. Kugi. Stability and incremental improvement of suboptimal MPC without terminal constraints. IEEE Trans. Automat. Contr., $55(11): 2576-2580,2010$.

[21] M. Granat, A. Ferguson, B. Andrews, and M. Delargy. The role of functional electrical stimulation in the rehabilitation of patients with incomplete spinal cord injury - observed benefits during gait studies. Spinal Cord, 31(4):207-215, 1993.

[22] K. Ha, S. Murray, and M. Goldfarb. An approach for the cooperative control of FES with a powered exoskeleton during level walking for persons with paraplegia. IEEE Trans. Neural Syst. Rehabil. Eng., 2015.

[23] K. Ha, H. Quintero, R. Farris, and M. Goldfarb. Enhancing stance phase propulsion during level walking by combining FES with a powered exoskeleton for persons with paraplegia. In Annual International Conference of the IEEE EMBC, pages 344-347, 2012.

[24] E. Hardin, R. Kobetic, L. Murray, M. Corado-Ahmed, G. Pinault, J. Sakai, S. Bailey, C. Ho, and R. Triolo. Walking after incomplete spinal cord injury using an implanted FES system: a case report. Journal of Rehabilitation research and Development, 44(3):333-346, 2007.

[25] B. Käpernick and K. Graichen. The gradient based nonlinear model predictive control software GRAMPC. In IEE Eur. Control Conf., pages 11701175, 2014.

[26] B. Käpernick and K. Graichen. GRAMPC - A gradient based MPC software for real-time applications, 2015.

[27] N. Kirsch, N. Alibeji, W. Dixon, and N. Sharma. A nonlinear control method to compensate for muscle fatigue during neuromuscular electrical stimulation. IEEE Transactions on Robotics, Submitted.

[28] N. Kirsch, N. Alibeji, and N. Sharma. Model predictive control-based dynamic control allocation in a hybrid neuroprosthesis. In ASME Dynamic Systems and Control Conference, San Antonio, Texas, 2014.

[29] K. Klose, P. Jacobs, J. Broton, R. Guest, B. Needham-Shropshire, N. Lebwohl, M. Nash, and B. Green. Evaluation of a training program for persons with SCI paraplegia using the parastep 1 ambulation system: part 1. ambulation performance and anthropometric measures. Arch. Phys. Med. Rehabil., 78(8):789-93, 1997.

[30] R. Kobetic, R. Triolo, and E. Marsolais. Muscle selection and walking performance of multichannel FES systems for ambulation in paraplegia. IEEE Trans. Rehabil. Eng., 5(1):23-29, 1997. 
[31] A. Kralj and T. Bajd. Functional electrical stimulation: standing and walking after spinal cord injury. CRC, 1989.

[32] F. Le, I. Markovsky, C. Freeman, and E. Rogers. Recursive identification of Hammerstein systems with application to electrically stimulated muscle. Control Eng. Pract., 20(4):386-396, 2012.

[33] E. Marsolais and R. Kobetic. Functional electrical stimulation for walking in paraplegia. The Journal of Bone and Joint Surgery, 69(5):728-733, 1987.

[34] D. Mayne, E. Kerrigan, E. van Wyk, and P. Falugi. Tube-based robust nonlinear model predictive control. Int. J. Robust Nonlinear Control, 21(11):1341-1353, 2011.

[35] L. Mendell and E. Henneman. Terminals of single Ia fibers: location, density, and distribution within a pool of 300 homonymous motoneurons. Journal of Neurophysiology, 34(1):171-187, 1971.

[36] S. Mohammed, P. Poignet, P. Fraisse, and D. Guiraud. Toward lower limbs movement restoration with input-output feedback linearization and model predictive control through functional electrical stimulation. Control Eng. Pract., 20(2):182-195, 2012.

[37] D. Popović, R. Stein, M. Oğuztöreli, M. Lebiedowska, and S. Jonić. Optimal control of walking with functional electrical stimulation: a computer simulation study. IEEE Trans. Rehabil. Eng., 7(1):69-79, 1999.

[38] H. Quintero, R. Farris, W. Durfee, and M. Goldfarb. Feasibility of a hybridFES system for gait restoration in paraplegics. In Annual International Conference of the IEEE EMBC, pages 483-486, 2010.

[39] H. Quintero, R. Farris, K. Ha, and M. Goldfarb. Preliminary assessment of the efficacy of supplementing knee extension capability in a lower limb exoskeleton with FES. In IEEE Eng. Med. Biol. Soc., volume 2012, pages 3360-3, 2012.

[40] R. Riener, J. Quintern, and G. Schmidt. Biomechanical model of the human knee evaluated by neuromuscular stimulation. J. Biomech., 29:1157-1167, 1996.

[41] I. Ross. A Primer on Pontryagin's Principle in Optimal Control. Collegiate Publishers, 2009.

[42] T. Schauer, N. O. Negard, F. Previdi, K. J. Hunt, M. H. Fraser, E. Ferchland, and J. Raisch. Online identification and nonlinear control of the electrically stimulated quadriceps muscle. Control Eng. Pract., 13:12071219, 2005.

[43] E. Schearer, Y. Liao, E. Perreault, M. Tresch, W. Memberg, R. Kirsch, and K. Lynch. Multi-muscle FES force control of the human arm for arbitrary goals. IEEE Trans. Neural Syst. Rehabil. Eng., 22(3):654-663, 2014. 
[44] N. Sharma, S. Bhasin, Q. Wang, and W. E. Dixon. Predictor-based control for an uncertain euler-lagrange system with input delay. Automatica, 47(11):2332-2342, 2011.

[45] N. Sharma, V. Mushahwar, and R. Stein. Dynamic optimization of FES and orthosis-based walking using simple models. IEEE Trans. Neural Syst. Rehabil. Eng., 22(1):114-126, 2014.

[46] N. Sharma, K. Stegath, C. M. Gregory, and W. E. Dixon. Nonlinear neuromuscular electrical stimulation tracking control of a human limb. IEEE Trans. Neural Syst. Rehabil. Eng., 17(6):576-584, 2009.

[47] R. Stein, E. Zehr, M. Lebiedowska, D. Popovic, A. Scheiner, and H. Chizeck. Estimating mechanical parameters of leg segments in individuals with and without physical disabilities. IEEE Trans. Rehabil. Eng., 4(3):201-211, 1996.

[48] P. Veltink, H. Chizeck, P. Crago, and A. El-Bialy. Nonlinear joint angle control for artificially stimulated muscle. IEEE Trans. Biomed. Eng., 39(4):368-80, 1992.

[49] Q. Wang, N. Sharma, M. Johnson, C. Gregory, and W. Dixon. Adaptive inverse optimal neuromuscular electrical stimulation. IEEE Trans. Cybern., 43(6):1710, 2013.

[50] S. Zhou, D. Lawson, W. Morrison, and I. Fairweather. Electromechanical delay in isometric muscle contractions evoked by voluntary, reflex and electrical stimulation. Eur. J. Appl. Physiol. Occup. Physiol., 70(2):138-145, 1995 . 